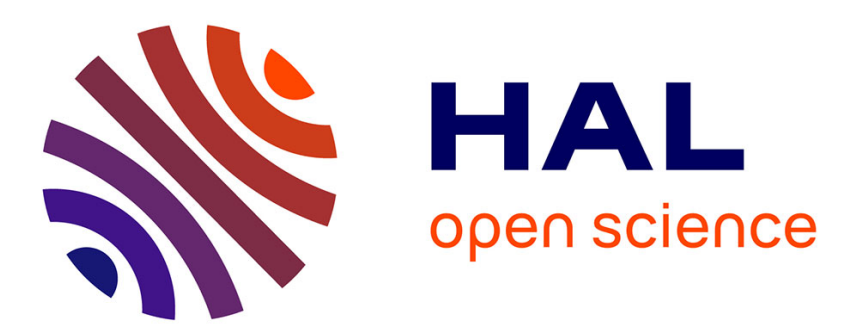

\title{
Adaptive trajectory control of off-road mobile robots: A multi-model observer approach
}

Mathieu Deremetz, Roland Lenain, Benoit Thuilot, Vincent Rousseau

\section{To cite this version:}

Mathieu Deremetz, Roland Lenain, Benoit Thuilot, Vincent Rousseau. Adaptive trajectory control of off-road mobile robots: A multi-model observer approach. IEEE International Conference on Robotics and Automation (ICRA), May 2017, Singapour, Singapore. pp.4407 - 4413, 10.1109/ICRA.2017.7989509 . hal-01658523

\section{HAL Id: hal-01658523 \\ https://hal.uca.fr/hal-01658523}

Submitted on 16 May 2020

HAL is a multi-disciplinary open access archive for the deposit and dissemination of scientific research documents, whether they are published or not. The documents may come from teaching and research institutions in France or abroad, or from public or private research centers.
L'archive ouverte pluridisciplinaire HAL, est destinée au dépôt et à la diffusion de documents scientifiques de niveau recherche, publiés ou non, émanant des établissements d'enseignement et de recherche français ou étrangers, des laboratoires publics ou privés. 


\title{
Adaptive trajectory control of off-road mobile robots: A multi-model observer approach
}

\author{
Mathieu Deremetz ${ }^{1}$, Roland Lenain ${ }^{1}$, Benoit Thuilot $^{2,3}$, and Vincent Rousseau ${ }^{1}$
}

\begin{abstract}
In this paper, the problems associated with accurate path tracking control in off-road conditions is addressed with model-based adaptive control. In particular, the estimation of grip conditions is investigated through the derivation of a new observer and by gathering kinematic and dynamic models into a single framework. This new reference point employs a unique observer regardless of the velocity of the robots. Previous approaches necessitated the switching of models depending upon the phenomena encountered as well as robot dynamics. The observer proposed here allows an accurate and reactive estimation of sliding. This permits to feed relevantly a control law based on an extended kinematic model, enabling accurate path tracking, even in harsh conditions and when facing significant dynamic effects such as spin around.
\end{abstract}

\section{INTRODUCTION}

Multiple industries, ranging from defense to agriculture, may benefit from the development of off-road autonomous mobile robotics. Such devices can efficiently and effectively help men and women to achieve painful or hazardous tasks. As a result, off-road applications are currently the object of intensive research, especially in the field of agriculture. These applications imply the implementation of actions (e.g., terrain crossing, posture stabilization, path tracking) submitted to very harsh and variable conditions. Despite these ever-changing and difficult grip conditions, robots have to be stable and accurate to ensure efficiency. This is not guaranteed by classical approaches [16], since they rely on the assumption of pure rolling without sliding conditions for different kinds of robots based on the classification of models introduced in [4]. These concepts are therefore appropriate when a robot moves on an almost flat, urban ground surface at low speed but are inaccurate on slippery ground [9].

Therefore, to increase the accuracy of path tracking in natural environments or in harsh conditions, new kinds of controls must be designed to account for varying grip conditions. Several approaches have been proposed in the framework of path following. First, sliding effects can be considered as a perturbation [1], which can be rejected with robust control techniques [15]. This effectively avoids the modeling of grip conditions, but often appears conservative. Another way to tackle sliding is to employ a model-based adaptive approach. This can be achieved by using extended

1 Irstea, Technologies and Information Support System Research Unit, 9 avenue Blaise Pascal, CS 20085, 63178 Aubière, France firstname. lastnamedirstea.fr

2 Clermont Université, Université Blaise Pascal, Institut Pascal, BP 10448, 63000 Clermont-Ferrand, France firstname. lastname@univ-bpclermont. fr

${ }^{3}$ CNRS, UMR 6602, Institut Pascal, 63178 Aubière, France kinematic models at low speeds, such as in [3] for carlike mobile robots and in [19] for a generalized scenario. However, such models and their associated observers are poorly suited for high speeds, since dynamic effects cannot be neglected. As a result, dynamic models need to be introduced. This has been proposed in previous work [14] using a model based on a partial dynamic model. Nevertheless, if the robot has to stop during its trajectory, the sliding observer associated with these models is irrelevant. It imposes to implement a speed threshold in algorithms to stop sliding estimation close to zero speed. Consequently, path following displays a lack of accuracy when the robot is forced to start and stop. The latter observer is also a concatenation of three estimation methods, which require more computing time to deliver the observation of the sliding.

In this paper, a new adaptive approach is proposed. It integrates an extended kinematic model and a dynamic model, and permits an estimation of sideslip angles independent from the robot velocity and ground surface variations. These estimations are then used to feed the adaptive and predictive control law proposed in [6] to control the steering angle of the front wheels of the robot and allow it to follow a path with a possible desired lateral offset.

This paper is composed as follows. First, the extended kinematic and dynamic models are recalled. The second part details previous work concerning the observation of lateral grip conditions, while the third section describes the proposed observer based on a multi-model. Then, the steering angle control is recalled from previous work. Finally, fullscale experiments are conducted to demonstrate the results and the efficiency of the proposed approach.

\section{MODELING}

\section{A. Mobile robot modeling}

The ambition of the proposed application is to allow for a mobile robot to follow a previously defined trajectory (i.e., computed or previously learned). This trajectory may include turns and ground variations such as slopes as well as grip condition modifications. To ensure accurate tracking, it is appropriate to use a model that takes into account the entire kinematics and dynamics of the robot.

In this paper, the mobile robot is a two-wheel drive vehicle. As is commonly perceived, the robot is viewed as a bicycle, with an equivalent front steering angle $\delta_{F}$ and a wheelbase $L$ (Figure 1). The robot speed $v$ is defined as the speed of the rear axle. This kind of model is commonly used in the field of mobile robotics, but generally supposes that the rolling without sliding condition is satisfied. In an off-road 
context,however, the assumption of rolling without sliding cannot be satisfied and introduces a tracking error when a control based on such a model is utilized. To overcome this phenomenon and consider non-ideal grip conditions in this model, two additional variables, $\beta_{F}$ and $\beta_{R}$, are added. These variables, called sideslip angles, denote the front and rear angles, respectively, between the tire orientation and the current speed vector orientation at the contact points $F$ and $R$. Thus, to follow the previously defined trajectory $\Gamma$, state variables of the robot are defined as follows:

$\diamond s$, the robot curvilinear abscissa. It is the curvilinear distance along $\Gamma$ of point $M$, the point on $\Gamma$ that is the closest to $R$. The curvature of $\Gamma$ at point $M$ is denoted $c(s)$.

$\diamond y$, the robot tracking error or lateral deviation. It is the algebraic distance between $R$ and $M$.

$\diamond \tilde{\theta}$, the robot angular error or angular deviation. It is the angle between the absolute robot heading, denoted $\theta$, and the orientation of the tangent to the trajectory at point $M$, denoted $\theta_{\text {tan }}$.

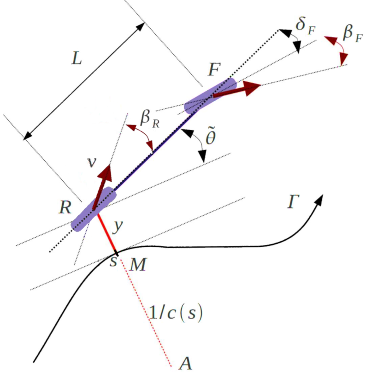

Fig. 1: Extended kinematic model of the robot with respect to reference trajectory $\Gamma$

Such a kinematic model is not sufficient in high dynamic conditions; it remains representative of circumstances utilizing low- and medium-speed path tracking with large curvature on nearly flat surfaces. However, when high speeds are considered, dynamical phenomena cannot be neglected; thus, this kinematic model cannot describe accurately robotic motion. To go further and preserve model accuracy at high speeds, an additional model, defined in Figure 2 and based on dynamic equations, is considered. This model, which acts as an addition to the extended kinematic model, possesses a description of the sideslip angles $\beta_{F}$ and $\beta_{R}$.

Integrating dynamic phenomena necessitates the location of the center of mass $G$. This center of mass is positioned along the robot centerline through two parameters, $L_{F}$ and $L_{R}$, which denote the front and rear wheelbases, respectively. It is assumed that the whole load, which relies on the mass $m$ and the gravity $g$, is applied at this point $G$. The speed of the center of mass is denoted $v_{G}$, and the robot moment of inertia around the vertical axis $(Z)$ is denoted $I_{z}$.

The robot is still perceived as a bicycle; consequently, only the two lateral contact forces $F_{F}$ and $F_{R}$ must be defined, which represent the lateral contact forces applied to the front and rear axles, respectively. When the robot moves on a slope, the gravity applies a force of $m g \sin (\alpha)$ on the sideway, where $\alpha$ denotes the roll angle of the chassis. Since only the path tracking is considered, longitudinal influences of this force as well as longitudinal contact forces are neglected. Thus, dynamic theorems lead to state variables defined as follows:

$\diamond \beta$, the global sideslip angle of the robot. It is the angle between the orientation of the speed vector at $G\left(v_{G}\right)$ and the absolute robot heading.

$\diamond \dot{\theta}$, the yaw speed of the robot. It is the angular speed of the robot chassis around the vertical axis $Z$.

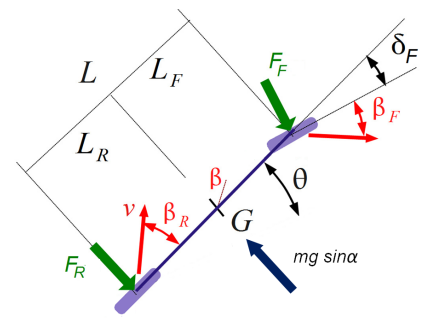

Fig. 2: Dynamic model of the robot

To solve this dynamic model, the lateral forces $F_{F}$ and $F_{R}$ must be estimated. For this purpose, many contact models may be considered, such as those discussed in [17], [10] and [11]. A common thread in all tire/road models is the relationship between force and the sideslip angle. However, this empirical model requires the prior knowledge of many parameters that strongly influence the trend of the curve. Despite this drawback, it should be noted that the global shape is always the same. Two sections can be distinguished: the first is a pseudo-slide zone for low sideslip angles, and the second is a constant overload zone for high sideslip angles.

To avoid the use of many parameters, which depend on the natural context and are difficult to identify, the following linear model (1) is considered. Two coefficients, $C_{F}$ and $C_{R}$, are defined, respectively, for the front and rear cornering stiffnesses.

$$
\left\{\begin{array}{l}
F_{F}=C_{F}(.) \beta_{F} \\
F_{R}=C_{R}(.) \beta_{R}
\end{array}\right.
$$

To obtain a coherent contact model, it is essential to take into account the non-linear variations of the ground conditions and the variation of properties due to vertical load and ground modifications. Thus, each cornering stiffness is considered to be variable so that a representative dynamic model can be obtained. The contact then becomes representative of non-linear behaviors (even if the sideslip angles are significant), and enables the consideration of variations in the grip conditions. This on-line adaptation, ensuring the model representation, is considered in section III.

Eventually, a combined model is obtained and used for the purpose of determining the parallel kinematics and dynamics of the robot. Details about the kinematics and dynamic equations are mentioned in the following subsections. 


\section{B. Location and deviation equations with respect to the} reference trajectory

According to classical kinematic analyses, such as is presented in [12], derivatives of the robot kinematic state variables (2) introduced above can be written as follows:

$$
\left\{\begin{aligned}
\dot{s}= & v \frac{\cos \left(\tilde{\theta}+\beta_{R}\right)}{1-c(s) y} \\
\dot{y}= & v \sin \left(\tilde{\theta}+\beta_{R}\right) \\
\dot{\tilde{\theta}}= & \dot{\theta}-\dot{\theta}_{\text {tan }}=v \cos \left(\beta_{R}\right) \frac{\tan \left(\delta_{F}+\beta_{F}\right)-\tan \left(\beta_{R}\right)}{L}-\ldots \\
& \ldots v \frac{c(s) \cos \left(\tilde{\theta}+\beta_{R}\right)}{1-c(s) y}
\end{aligned}\right.
$$

This model is defined if the $1-c(s) y \neq 0$ condition is true, which means that the center of curvature $A$ should not be superimposed with the center of the rear axle $R$. However, if the robot is properly initialized, such a case is never reached in practice.

\section{Dynamic equations}

Similarly, according to classical dynamic analyses, such as is presented in [13], derivatives of the robot dynamic state variables (3) and related variables (4) introduced above can be written as follows:

$$
\left\{\begin{aligned}
\ddot{\theta}= & \frac{1}{I_{z}}\left(L_{R} C_{R} \beta_{R}-L_{F} C_{F} \beta_{F} \cos \left(\delta_{F}\right)\right) \\
\frac{d\left(v_{G} \sin (\beta)\right)}{d t}= & \frac{\left(C_{F} \beta_{F} \cos \left(\delta_{F}\right)+C_{R} \beta_{R}\right)}{m}-\ldots \\
& \ldots g \sin (\alpha)+v \cos \left(\beta_{R}\right) \dot{\theta}
\end{aligned}\right.
$$

In addition:

$$
\left\{\begin{array}{l}
\beta=\arctan \left(\frac{L_{R} \tan \left(\beta_{F}+\delta_{F}\right)+L_{F} \tan \left(\beta_{R}\right)}{L}\right) \\
v_{G}=\frac{v \cos \left(\beta_{R}\right)}{\cos (\beta)}
\end{array}\right.
$$

The derivative of the state variable $v_{G} \sin (\beta)$ is highly recommended in its given form to avoid division by zero (induced by a zero speed) which results in a singularity and disrupts the continuity of the algorithms. This formula is used in section IV to define the evolution model of the proposed observer.

\section{PREVIOUS ESTIMATION OF SIDESLIP ANGLES AND CORNERING STIFFNESSES}

To obtain accurate path tracking based on an adaptive and predictive control law, which is detailed in section $\mathrm{V}$, it is compulsory to retrieve real-time values for the sideslip angles, $\beta_{F}$ and $\beta_{R}$, and the cornering stiffnesses, $C_{F}$ and $C_{R}$, regardless of the dynamic variations of the robot. However, the direct measure of these variables is hardly feasible using sensors. As a consequence, these values must be indirectly estimated.

Such estimations, which have been previously developed [14], require three observation steps to obtain suitable and reactive values. First, the sideslip angle $\beta$ is computed through the first line of (4) using estimations of the sideslip angles based on an extended kinematic model [12]. Subsequently, an observer, which is based on the dynamic equations (3), is used to compute the lateral forces $F_{F}$ and $F_{R}$. From these estimations, and by relying on (1) in conjunction with the calculation method known as the MIT Rule [2], it is then possible to obtain suitable estimations for the cornering stiffnesses. Finally, an algorithm estimates the dynamic states of the mobile robot to readjust the kinematic sideslip angles into dynamic ones and improve reactivity. Nevertheless, a singularity appears when the robot stops, and instability at low speeds may occur because of division by the value of the speed in the linearized models used in each observer algorithm.

To avoid estimation issues when the speed is null or low, a kinematic observer described in [8] is proposed. Its evolution model is based on (2), and may be written as follows:

$$
\dot{\xi}=\left[\begin{array}{c}
\dot{\xi}_{d e v} \\
\dot{\xi}_{\beta_{i}}
\end{array}\right]=\left[\begin{array}{c}
f\left(\xi_{d e v}, \xi_{\beta_{i}}, v, \delta\right) \\
0_{2 \times 1}
\end{array}\right]
$$

where $\xi$ is split into two sub-states:

$\diamond \xi_{\text {dev }}=\left[\begin{array}{ll}y & \tilde{\theta}\end{array}\right]^{T}$, which constitutes the deviations of the robot with respect to the trajectory $\Gamma$.

$\diamond \xi_{\beta_{i}}=\left[\begin{array}{ll}\beta_{F} & \beta_{R}\end{array}\right]^{T}$, which is composed of the sideslip angles, to be estimated.

and where $f\left(\xi_{\text {dev }}, \xi_{\beta_{i}}, v, \delta\right)$ is directly deduced from the last two lines of model (2).

Hereafter, $\xi$ should be considered as an effectively measured variable, while $\hat{\xi}$ should be considered as an observed or estimated variable. $\tilde{\xi}$ is defined as the observation error $\tilde{\xi}=\xi-\hat{\xi}$.

In consideration of the candidate Lyapunov function (6), which is the norm of the state $\xi$, it has been shown in [8] that the whole observer state $\hat{\xi}$ converges to the actual state $\xi$. Moreover, the structure of this observer does not necessitate the linearization of the extended kinematic model and consequently avoids singularities close to zero speed.

$$
V=\frac{1}{2} K_{\beta} \tilde{\xi}_{\text {dev }}^{T} \tilde{\xi}_{\text {dev }}+\frac{1}{2} \tilde{\xi}_{\beta_{i}}^{T} \tilde{\xi}_{\beta_{i}}
$$

However, this observer does not take into account the dynamic behavior of the robot. Consequently, its estimated values are not relevant when the robot suffers important dynamic effects such as those experienced at high speed or on a tilted path.

It is therefore relevant to design an observer which is able to estimate reactive values of the lateral effects with an optimized computing time regardless of the dynamic phenomena and that can avoid a singularity at zero speed. Such an observer is proposed in the following section. 


\section{PROPOSED OBSERVER}

In this section, a new one-step observer is proposed and is developed based on the observer structure described previously in [8]. The major feature of the proposed observer is the integration of kinematic and dynamic models into a common state vector with the ambition of instantly obtaining suitable and reactive estimations of sideslip angles and cornering stiffnesses. By using a relevant state space model, this observer also permits estimations regardless of the speed of the robot.

For this purpose, let us consider the state vector $\xi$ defined as follows:

$$
\xi=\left[\begin{array}{c}
\xi_{d e v} \\
\xi_{d y n} \\
\xi_{\beta_{i}} \\
\xi_{C_{i}}
\end{array}\right]
$$

The state vector $\xi$ is further split into four sub-states:

$\diamond \xi_{d e v}$ and $\xi_{\beta_{i}}$ are the same as in (5).

$\diamond \xi_{d y n}=\left[\dot{\theta}\left(v_{G} \sin (\beta)\right)\right]^{T}$, which constitutes the dynamic variables of the robot.

$\diamond \xi_{C_{i}}=\left[\begin{array}{ll}C_{F} & C_{R}\end{array}\right]^{T}$, which is composed of the cornering stiffnesses, to be estimated.

In this state space description, only $\xi_{\text {dev }}$ and $\dot{\theta}$ are supposed to be measured, as highlighted in section V. Moreover, the steering angle $\delta_{F}$, and the velocity $v$ are measured and considered as variable parameters. Since the proposed observer supplies the value of $\hat{\xi}_{\beta_{i}}$, an estimation of $v_{G} \sin (\beta)$ is made possible thanks to (4) and is considered as a virtual measure even if it is retrieved as a consequence of estimated values. This is possible due to the choice for observer gains defining its dynamics. Indeed, the convergence for $\hat{\xi}_{\beta_{i}}$ is chosen faster than for $\hat{\xi}_{d y n}$, and can then be assumed to be a measured value. As a result, in the following section, the two sub-states $\xi_{d e v}$ and $\xi_{d y n}$ are supposed to be measured.

\section{A. Observer equations}

Equations for the proposed observer are as follows:

$$
\dot{\hat{\xi}}=\left[\begin{array}{c}
\dot{\hat{\xi}}_{\text {dev }} \\
\dot{\hat{\xi}}_{d y n} \\
\dot{\hat{\xi}}_{\beta_{i}} \\
\dot{\hat{\xi}}_{C_{i}}
\end{array}\right]=\left[\begin{array}{c}
f\left(\xi_{\text {dev }}, \hat{\xi}_{\beta_{i}}, v, \delta\right)+\alpha_{d e v}\left(\tilde{\xi}_{d e v}\right) \\
g\left(\hat{\xi}_{\text {dyn }}, \hat{\xi}_{\beta_{i}}, \hat{\xi}_{C_{i}, v}, \delta\right)+\alpha_{d y n}\left(\tilde{\xi}_{d y n}\right) \\
\alpha_{\beta_{i}}\left(\tilde{\xi}_{d e v}, \tilde{\xi}_{d y n}\right) \\
\alpha_{C_{i}}\left(\tilde{\xi}_{d y n}\right)
\end{array}\right]
$$

The functions $f\left(\xi_{d e v}, \hat{\xi}_{\beta_{i}}, v, \delta\right)$ and $g\left(\xi_{d y n}, \hat{\xi}_{\beta_{i}}, \hat{\xi}_{C_{i}}, v, \delta\right)$ are directly deduced from the last two lines of models (2) and (3). $\alpha_{d e v}, \alpha_{d y n}, \alpha_{\beta_{i}}$ and $\alpha_{C_{i}}$ are functions that must be judiciously chosen to ensure the convergence of $\tilde{\xi}$ to zero. The ultimately estimated sub-states $\hat{\xi}_{\beta_{i}}$ and $\hat{\xi}_{C_{i}}$ will be considered as suitable estimations for the robot lateral dynamics.

There are no available equations related to sideslip angles and cornering stiffness evolutions, $\dot{\beta_{F}}, \dot{\beta_{R}}, \dot{C}_{F}$ and $\dot{C}_{R}$. Consequently, $\dot{\xi}_{\beta_{i}}$ and $\dot{\xi}_{C_{i}}$ are each set to $0_{2 \times 1}$.

\section{Proposition:}

The choice (9) for feedback functions introduced in (8) ensures the convergence of $\hat{\xi} \rightarrow \xi$. In particular, it permits the estimation of sideslip angles for their actual but not measured values.

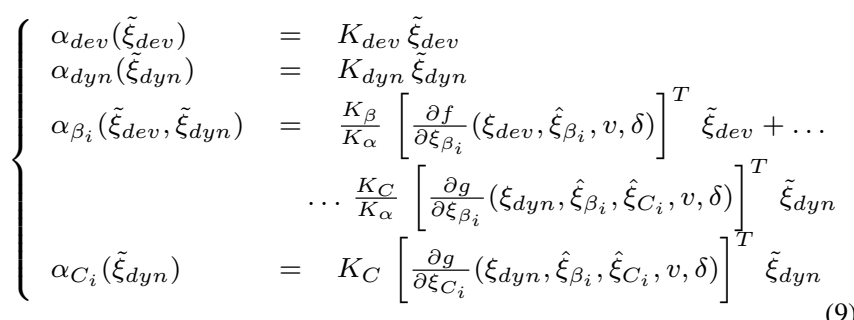

such as:

$\diamond K_{d e v}$ and $K_{d y n}$ are each $2 \times 2$ positive diagonal matrices.

$\diamond K_{\beta}, K_{C}$ and $K_{\alpha}^{1}$ are positive scalars.

\section{B. Proof}

From (8), the derivative of $\tilde{\xi}$ can be computed as:

$$
\begin{gathered}
\dot{\tilde{\xi}}=\quad\left[\begin{array}{c}
\dot{\tilde{\xi}}_{\text {dev }} \\
\dot{\tilde{\xi}}_{\text {dyn }} \\
\dot{\tilde{\xi}}_{\beta_{i}} \\
\dot{\tilde{\xi}}_{C_{i}}
\end{array}\right] \\
=\left[\begin{array}{c}
f\left(\xi_{d e v}, \xi_{\beta_{i}}, v, \delta\right)-f\left(\xi_{d e v}, \hat{\xi}_{\beta_{i}}, v, \delta\right)-\ldots \\
\ldots\left(\alpha_{d e v}\left(\tilde{\xi}_{d e v}\right)\right. \\
\left.g, \xi_{\beta_{i}}, \xi_{C_{i}}, v\right)-g\left(\xi_{d y n}, \hat{\xi}_{\beta_{i}}, \hat{\xi}_{C_{i}}, v, \delta\right)-\ldots \\
\ldots \alpha_{d y n}\left(\tilde{\xi}_{d y n}\right) \\
-\alpha_{\beta_{i}}\left(\tilde{\xi}_{\text {dev }}, \tilde{\xi}_{\text {dyn }}\right) \\
-\alpha_{C_{i}}\left(\tilde{\xi}_{d y n}\right) \\
\end{array}\right]
\end{gathered}
$$

Considering the linearization of function $f\left(\xi_{\text {dev }}, \xi_{\beta_{i}}, v, \delta\right)$ around the estimated sideslip angles $\hat{\xi}_{\beta_{i}}$, $f$ can be rewritten as follows:

$$
\begin{gathered}
f\left(\xi_{\text {dev }}, \xi_{\beta_{i}}, v, \delta\right)= \\
f\left(\xi_{\text {dev }}, \hat{\xi}_{\beta_{i}}, v, \delta\right)+\frac{\partial f}{\partial \xi_{\beta_{i}}}\left(\xi_{\text {dev }}, \hat{\xi}_{\beta_{i}}, v, \delta\right) \tilde{\xi}_{\beta_{i}}+O\left(\tilde{\xi}_{\beta_{i}}^{2}\right)
\end{gathered}
$$

Considering the linearization of function $g\left(\xi_{d y n}, \xi_{\beta_{i}}, \xi_{C_{i}}, v, \delta\right)$ around the estimated sideslip angles $\hat{\xi}_{\beta_{i}}$ and the cornering stiffnesses $\hat{\xi}_{C_{i}}, g$ can be rewritten as follows:

$$
\begin{gathered}
g\left(\xi_{d y n}, \xi_{\beta_{i}}, \xi_{C_{i}}, v, \delta\right)= \\
g\left(\xi_{d y n}, \hat{\xi}_{\beta_{i}}, \hat{\xi}_{C_{i}}, v, \delta\right)+\frac{\partial g}{\partial \xi_{\beta_{i}}}\left(\xi_{d y n}, \hat{\xi}_{\beta_{i}}, \hat{\xi}_{C_{i}}, v, \delta\right) \tilde{\xi}_{\beta_{i}}+\ldots \\
\ldots \frac{\partial g}{\partial \xi_{C_{i}}}\left(\xi_{d y n}, \hat{\xi}_{\beta_{i}}, \hat{\xi}_{C_{i}}, v, \delta\right) \tilde{\xi}_{C_{i}}+O\left(\tilde{\xi}_{\beta_{i}}^{2}, \tilde{\xi}_{C_{i}}^{2}\right)
\end{gathered}
$$

One then acquires the following observation error dynamics:

\footnotetext{
${ }^{1} K_{\alpha}$ allows the balancing of $K_{\beta}$ and $K_{C}$ and then balances the influence of kinematics and dynamics on sideslip estimations
} 


$$
\begin{gathered}
\dot{\tilde{\xi}}=\quad\left[\begin{array}{c}
\dot{\tilde{\xi}}_{\text {dev }} \\
\dot{\tilde{\xi}}_{d y n} \\
\dot{\tilde{\xi}}_{\beta_{i}} \\
\dot{\tilde{\xi}}_{C_{i}}
\end{array}\right] \\
=\left[\begin{array}{c}
\frac{\partial f}{\partial \xi_{\beta_{i}}}\left(\xi_{d e v}, \hat{\xi}_{\beta_{i}}, v, \delta\right) \tilde{\xi}_{\beta_{i}}-\alpha_{d e v}\left(\tilde{\xi}_{d e v}\right) \\
\frac{\partial g}{\partial \xi_{\beta_{i}}}\left(\xi_{d y n}, \hat{\xi}_{\beta_{i}}, \hat{\xi}_{C_{i}}, v, \delta\right) \tilde{\xi}_{\beta_{i}}+\ldots \\
\ldots \frac{\partial g}{\partial \xi_{C_{i}}}\left(\xi_{d y n}, \hat{\xi}_{\beta_{i}}, \hat{\xi}_{C_{i}}, v, \delta\right) \tilde{\xi}_{C_{i}}-\alpha_{d y n}\left(\tilde{\xi}_{d y n}\right) \\
-\alpha_{\beta_{i}}\left(\tilde{\xi}_{d e v}, \tilde{\xi}_{d y n}\right) \\
-\alpha_{C_{i}}\left(\tilde{\xi}_{d y n}\right)
\end{array}\right]
\end{gathered}
$$

Let us consider the candidate Lyapunov function, (14), defined from the whole observation error:

$V=\frac{1}{2} K_{\beta} \tilde{\xi}_{d e v}^{T} \tilde{\xi}_{d e v}+\frac{1}{2} K_{C} \tilde{\xi}_{d y n}^{T} \tilde{\xi}_{d y n}+\frac{1}{2} K_{\alpha} \tilde{\xi}_{\beta_{i}}^{T} \tilde{\xi}_{\beta_{i}}+\frac{1}{2} \tilde{\xi}_{C_{i}}^{T} \tilde{\xi}_{C_{i}}$

The derivative of (14) considering the solutions of system (13) is:

$$
\begin{array}{r}
\dot{V}=K_{\beta}\left[\frac{\partial f}{\partial \xi_{\beta_{i}}}\left(\xi_{d e v}, \hat{\xi}_{\beta_{i}}, v, \delta\right) \tilde{\xi}_{\beta_{i}}-\alpha_{d e v}\left(\tilde{\xi}_{\text {dev }}\right)\right]^{T} \tilde{\xi}_{\text {dev }}+\ldots \\
\ldots K_{C}\left[\frac{\partial g}{\partial \xi_{\beta_{i}}}\left(\xi_{d y n}, \hat{\xi}_{\beta_{i}}, \hat{\xi}_{C_{i}}, v, \delta\right) \tilde{\xi}_{\beta_{i}}+\ldots\right. \\
\left.\ldots \frac{\partial g}{\partial \xi_{C_{i}}}\left(\xi_{d y n}, \hat{\xi}_{\beta_{i}}, \hat{\xi}_{C_{i}}, v, \delta\right) \tilde{\xi}_{C_{i}}-\alpha_{d y n}\left(\tilde{\xi}_{d y n}\right)\right]^{T} \tilde{\xi}_{\text {dyn }}-\ldots \\
\ldots K_{\alpha} \tilde{\xi}_{\beta_{i}}^{T} \alpha_{\beta_{i}}\left(\tilde{\xi}_{d e v}, \tilde{\xi}_{d y n}\right)-\tilde{\xi}_{C_{i}}^{T} \alpha_{C_{i}}\left(\tilde{\xi}_{d y n}\right)
\end{array}
$$

After injecting the expressions in (9), the derivative function $\dot{V}$ can be shown to be:

$$
\dot{V}=-K_{\beta} K_{d e v} \tilde{\xi}_{d e v}^{T} \tilde{\xi}_{d e v}-K_{C} K_{d y n} \tilde{\xi}_{d y n}^{T} \tilde{\xi}_{d y n}
$$

which is negative, and implies that $\tilde{\xi}_{\text {dev }}$ and $\tilde{\xi}_{d y n}$ converge to zero. Hence, by using the Krasovskii-LaSalle theorem, $\tilde{\xi}_{\beta_{i}}$ and $\tilde{\xi}_{C_{i}}$ also converge to zero so long as the mobile robot is subjected to lateral effects. Then, $\hat{\xi}_{\beta_{i}} \rightarrow \xi_{\beta_{i}}$. Finally, these estimations can be used for robot control.

\section{CONTROL ALGORITHMS}

Since relevant values of $\hat{\beta_{F}}$ and $\hat{\beta_{R}}$ are available, model (2) is entirely known and may be used to process path tracking controls. To properly accomplish this objective, a front-steering adaptive control law has been used to ensure the convergence of lateral deviation $y$ to zero regardless of the speed. Moreover, to anticipate trajectory overshoots due to the actuator settling time, a predictive curvature servoing has been implemented. Only a few details about these control algorithms are given in this section, since this approach has been previously proposed in [18].

\section{A. Adaptive control algorithm}

As model (2) is structurally close to classical kinematic models, it can be transformed into a chained form. Exact linearization techniques can then be used, after which front steering control law can be obtained:

$$
\begin{gathered}
\delta_{F}=\arctan \left[\tan \left(\hat{\beta_{R}}\right)+\frac{L}{\cos \left(\hat{\beta_{R}}\right)}\left(\frac{c(s) \cos \gamma}{\alpha}+\frac{A \cos ^{3} \gamma}{\alpha^{2}}\right)\right]-\hat{\beta_{F}} \\
\text { with: }\left\{\begin{aligned}
\gamma & =\tilde{\theta}+\hat{\beta_{R}} \\
\alpha & =1-c(s) y \\
\eta & =\tan \gamma \\
A & =-K_{p} y-K_{d} \alpha \eta+c(s) \alpha \tan ^{2} \gamma
\end{aligned}\right.
\end{gathered}
$$

where $K_{p}$ and $K_{d}$ are positive gains that are homogeneous to proportional and differential actions.

\section{B. Predictive curvature servoing}

Next, the steering law (17) can be split into two terms, wherein predictive techniques can be applied to the first term:

$$
\delta_{F}=\delta_{\text {Traj }}^{\text {Pred }}+\delta_{\text {Deviation }}
$$

where,

$\diamond \delta_{\text {Traj }}^{\text {Pred depends primarily on curvature and ensures the }}$ equality between the trajectory and vehicle curvature. Since the reference path curvature can be anticipated, the term $\delta_{\text {Traj }}^{\text {Pred }}$ is designed from a model predictive control approach, making use of the future curvature of the reference path.

$\diamond \delta_{\text {Deviation }}$ ensures the convergence of the lateral deviation to zero from the previously measured lateral deviations and previously estimated grip conditions. It is straightforwardly computed from (17).

Details of this approach can be found in [6].

\section{SIMULATION AND EXPERIMENTAL RESULTS}

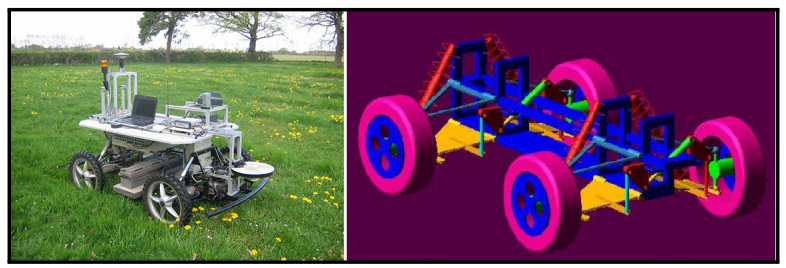

Fig. 3: Experimental platform and its simulator

TABLE I: Robot features

\begin{tabular}{|c||c|}
\hline Weight $(\mathrm{m})$ & $420 \mathrm{~kg}$ \\
\hline Wheelbase $(\mathrm{L})$ & $1.2 \mathrm{~m}$ \\
\hline Maximum steering angle & $20^{\circ}$ \\
\hline Steering angle response time & $0.4 \mathrm{~s}$ \\
\hline Maximum speed & $8 \mathrm{~m} . \mathrm{s}^{-1}$ \\
\hline
\end{tabular}

Making use of the proposed estimation strategy, the previous adaptive and predictive control algorithms have been tested using a MATLAB/ADAMS co-simulator and through full-scale experiments with the mobile robot depicted in Figure 3. This robot is electrical, is four-wheel drive and has a front steering axle. It is able to reach a maximum speed of $8 \mathrm{~m} . \mathrm{s}^{-1}$ and is equipped with an IMU and a RTK-GPS (2-centimeter accuracy at $10 \mathrm{~Hz}$ ), which are located at the 
middle of the rear axle. It has been specifically developed for testing algorithms dedicated to off-road low- and high-speed motion, and has the features described in Table I.

\section{A. Simulation Results}

Controlling a robot equipped with algorithms that possess singularities can be dangerous for men and may deteriorate the robot. As a consequence, it is relevant to compare the continuity of the proposed observer and the previous dynamic observer at low- and zero speed on a MATLAB/ADAMS co-simulator.

A trial was carried out at low speed $\left(3 \mathrm{~m}_{\mathrm{s}} \mathrm{s}^{-1}\right)$ on a flat surface with a stopping point in the middle of the trajectory. The results for the $3 \mathrm{~m} \cdot \mathrm{s}^{-1}$ trial are depicted in Figures 4, 5 and 6.

Figure 4 illustrates the mobile robot trajectory in blue and the proposed observer with respect to the reference path in black. Figures 5 and 6 demonstrate the front and the rear sideslip angle estimations of the robot for each observation case.

It is apparent in Figure 4 that the path tracking is not spoiled at low and zero speeds when the proposed observer is used. This is not the case for the previous dynamic observer, because a divergence of the sideslip angles can be noticed in Figures 5 and 6 when the speed of the robot is nearly zero (i.e., when the time is between 15 and 17 seconds). This divergence of the sideslip angles leads to a divergence of $\delta_{F}$. The result of the path following with the previous observer is not displayed in Figure 4 because the robot was unable to accomplish path tracking after the stopping point.

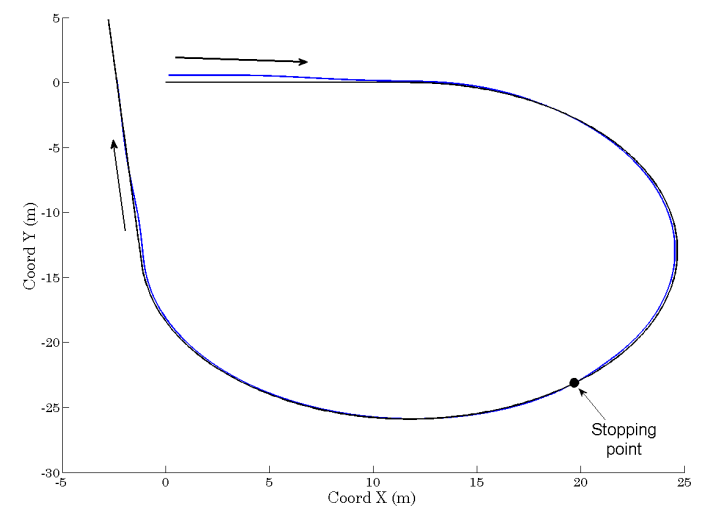

Fig. 4: $3 \mathrm{~m} . \mathrm{s}^{-1}$ path tracking results with stopping point

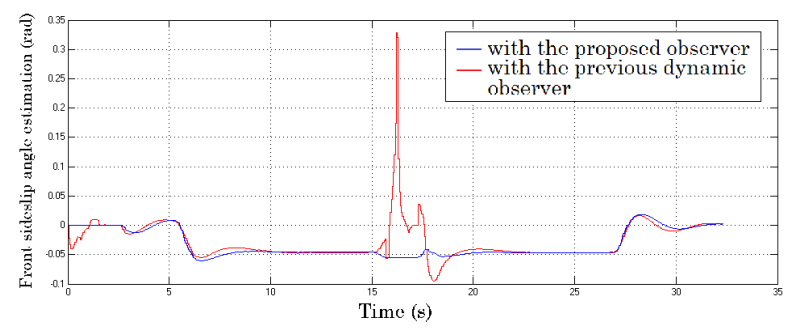

Fig. 5: $3 \mathrm{~m} . \mathrm{s}^{-1}$ rear sideslip angle estimation with stopping point

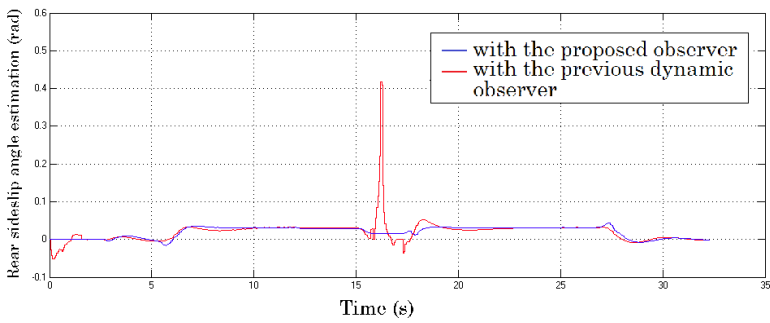

Fig. 6: $3 m \cdot s^{-1}$ front sideslip angle estimation with stopping point

\section{B. Experimental Results}

1) Path definition: To generate observable phenomena, the trajectory depicted in Figure 7 has been chosen as the path to be followed. This trajectory is relevant because it is composed of a set of tracking situations that test the proposed observer in each dynamic case:

$\diamond$ Case 1: straight line and S-bend on a flat surface, illustrated in Figure 8.1

$\diamond$ Case 2: bend on a surface with a varying slope (flat $\rightarrow$ 15-degree slope $\rightarrow$ flat), illustrated in Figure 8.2

$\diamond$ Case 3: straight line tracking on a 15-degree slope, illustrated in Figure 8.3

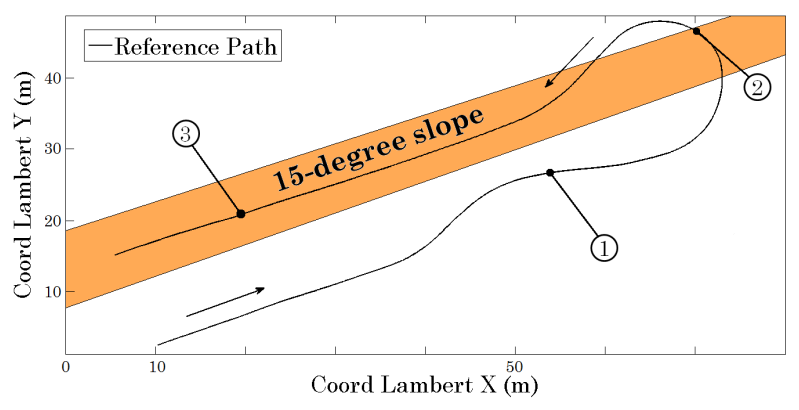

Fig. 7: Trajectory chosen for the experiments
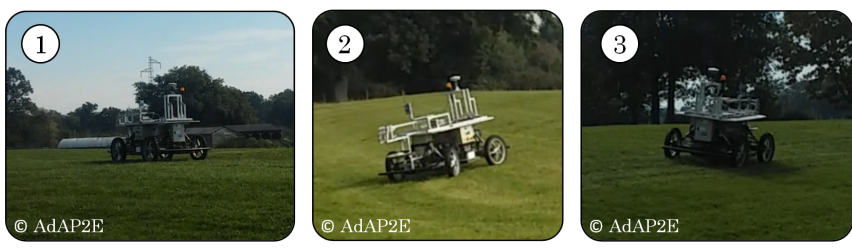

Fig. 8: Pictures of the robot during trials

2) Sideslip estimation and path tracking: A set of trials have been conducted at high speeds $\left(4-6 \mathrm{~m} \cdot \mathrm{s}^{-1}\right)$. The results for the $5 \mathrm{~m} \cdot \mathrm{s}^{-1}$-trial are depicted in Figures 9 and 10. For these trials, the values of the gains have been chosen as follows: $K_{y}=K_{\tilde{\theta}}=15, K_{\dot{\theta}}=K_{v \beta}=20, K_{\beta}=2.10^{12}$, $K_{\alpha}=5.10^{11}, K_{C}=5.10^{7}$.

Figure 9 shows the mobile robot trajectory with the proposed observer, the previous dynamic observer and without an observer. Figure 10 demonstrates the lateral deviations of the robot with respect to the trajectory for each observation case. 
It is apparent in Figures 9 and 10 that the path tracking is not spoiled at high speeds when the proposed observer is used instead of the previous dynamic observer. Indeed, lateral deviations are closely similar all along the trajectory, and do not exceed the thresholds defined by $\pm \frac{\text { speed }}{10}$, which validates the reliability of the tracking. Moreover, it can be observed that the non-implementation of a sliding observer harms the path tracking quality, especially when dynamic phenomena (bends and variable slopes) appear and when the speed increases.

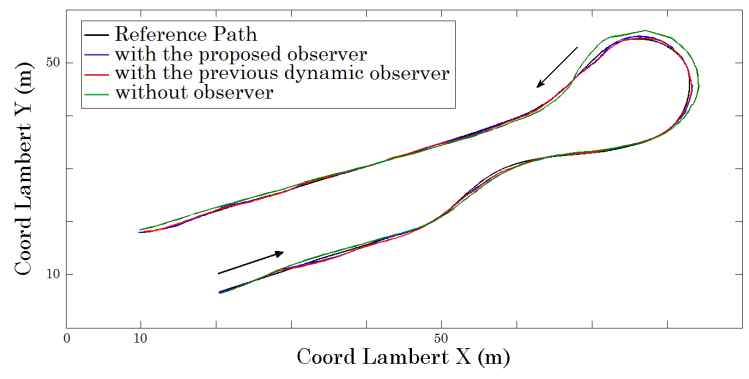

Fig. 9: $5 m . s^{-1}$ path tracking results

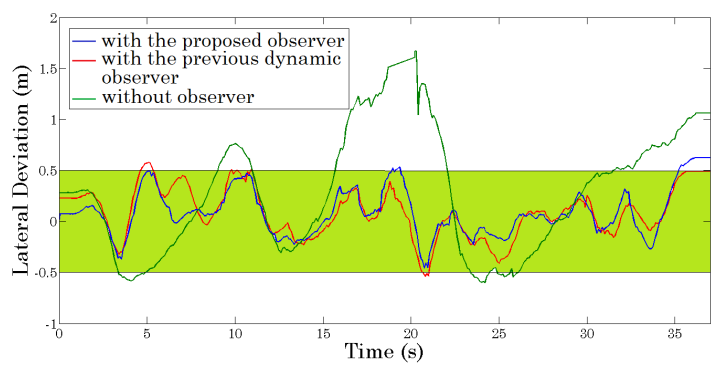

Fig. 10: $5 \mathrm{~m} . \mathrm{s}^{-1}$ lateral deviation results

\section{CONCLUSION}

This paper proposes a control strategy to be utilized for a mobile robot to follow a path in an off-road context. Because of harsh grip conditions and ground variations, classical models based on rolling without sliding assumptions cannot be used with satisfactory accuracy. The dynamic observer derived within previous research can supply efficient results but has to be stopped each time the robot speed approaches zero, leading to transient unsatisfactory behaviors when the robot is restarted. Here, a new modelbased adaptive approach is proposed to estimate on-line the lateral dynamics of the robot in harsh conditions with no singularity at zero speed. By integrating the dynamic model in observer equations, it has been proven that an estimation of sliding variations can be obtained for all of the range of speeds and ground geometries. The compact observer structure also saves computation time compared to other dynamic observers. As shown in the experimental section, the combination of the new observer with a predictive low obtains a more highly accurate path tracking regardless of the speed and the dynamic perturbations encountered in the natural environment. Future work will be focused on the implementation of a predictive algorithm that integrates parts of the adapted dynamic parameters $\hat{C}_{F}$ and $\hat{C}_{R}$ to further increase the accuracy of path tracking.

\section{ACKNOWLEDGMENT}

This work has received the support of French National Research Agency under the grant number ANR-14-CE270004 attributed to AdAP2E project and has been sponsored by the IMobS3 Laboratory of Excellence under the grant number ANR-10-LABX-16-01.

\section{REFERENCES}

[1] B. d'Andréa-Novel and G. Campion and G. Bastin (1995). Control of wheeled mobile robots not satisfying ideal velocity constraints: a singular perturbation approach. International Journal of Robust and Nonlinear Control, vol. 5, no. 4, pp. 243-267.

[2] K.J. Astrom and B. Wittenmark. Adaptive control (2nd edition). NewYork : Addidson-Wesley, 1994.

[3] G. Bayar, M. Bergerman and A. B Koku (2016). Improving the trajectory tracking performance of autonomous orchard vehicles using wheel slip compensation. Biosystems Engineering, vol. 146, pp. 149164.

[4] G. Campion and G. Bastin and B. d'Andréa-Novel (1993). Structural properties and classification of kinematic and dynamic models of wheeled mobile robots. In IEEE international conference on robotics and automation, pp. 462-469, Atlanta, Georgia (USA).

[5] C. Cariou and R. Lenain and B. Thuilot and P. Martinet (2008) Adaptive control of four-wheel-steering off-road mobile robots: Application to path tracking and heading control in presence of sliding. In IEEE/RSJ International Conference on Intelligent Robots and Systems (IROS), pp. 1759-1764, Nice (France).

[6] C. Cariou and R. Lenain. and B. Thuilot and M. Berducat (2009). Automatic guidance of a four-wheel-steering mobile robot for accurate field operations. Journal of Field Robotics, vol. 26, no. 6-7, pp. 504518.

[7] H. Fang and L. Dou and J. Chen and R. Lenain and B. Thuilot and P. Martinet (2011). Robust anti-sliding control of autonomous vehicles in presence of lateral disturbances. Control Engineering Practice, vol. 19, no. 5, pp. 468-478.

[8] A. Guillet and R. Lenain and B. Thuilot and P. Martinet (2014). Adaptable Robot Formation Control: Adaptive and Predictive Formation Control of Autonomous Vehicles. In IEEE Robotics Automation Magazine, vol. 21, no. 1, pp. 28-39.

[9] B. Innocenti and P. Ridao and N. Gascons and A. El-Fakdi and B. Lopez and J. Salvi (2004). Dynamical model parameters identification of a wheeled mobile robot. In IFAC/EURON symposium on intelligent autonomous vehicles, Lisboa (Portugal).

[10] K. Johanastrom and C. Canudas-de-Wit. Revisiting the LuGre friction model (2008). IEEE control Systems, vol. 28, no 6, p. 101-114.

[11] U. Kiencke and L. Nielsen. Vehicle modelling (2005). Automotive Control Systems: For Engine, Driveline, and Vehicle, p. 301-349.

[12] R. Lenain and B. Thuilot and C. Cariou and P. Martinet (2006). High accuracy path tracking for vehicles in presence of sliding: Application to farm vehicle automatic guidance for agricultural tasks. Autonomous Robots, vol. 21, no. 1, pp. 79-97.

[13] R. Lenain, B. Thuilot, C. Cariou, P. Martinet (2010). Mixed kinematic and dynamic sideslip angle observer for accurate control of fast offroad mobile robots. Journal of Field Robotics, vol. 27, no. 2, pp. 181-196.

[14] R. Lenain and B. Thuilot and O. Hach and P. Martinet (2011). Highspeed mobile robot control in off-road conditions: a multi-model based adaptive approach. In IEEE International Conference on Robotics and Automation (ICRA), pp. 6143-6149, Shanghai (China).

[15] J. Lu, S. Sekhavat, M. Xie and C. Laugier (2000). Sliding mode control for nonholonomic mobile robot. In Proceedings of the International Conference on Control, Automation, Robotics and Vision, ICARCV.

[16] P. Morin and C. Samson (2008). Motion control of wheeled mobile robots. Springer Handbook of Robotics, pp. 799-826.

[17] H.B. Pacejka (2002). Tire and vehicle dynamics. Society of Automotive Engineers, pp 627.

[18] C. Samson (1995). Control of chained systems. Application to path following and time-varying point stabilization of mobile robots. IEEE Transactions on Automatic Control, vol. 40, no. 1, pp. 64-77.

[19] D. Wang and C.B. Low (2007). An analysis of wheeled mobile robots in the presence of skidding and slipping: Control design perspective. In IEEE International Conference on Robotics and Automation (ICRA), pp. 2379-2384, Roma (Italy). 